\title{
Doença celíaca: repercussões na mineralização óssea
}

\author{
Celiac disease: effects on bone mineralization
}

\author{
Giselia Alves Pontes da Silva*
}

\begin{abstract}
A té recentemente, a doença celíaca (DC) era considerada rara; acreditava-se que ela afetava sobretudo as crianças, e que se manifestava clinicamente de forma exuberante e característica. Com o surgimento dos testes sorológicos específicos a partir de 1980, tornou-se possível a investigação de um maior número de pessoas através de screening populacional, sendo identificado um
\end{abstract} grande número de portadores de DC nas diversas faixas etárias. As formas clássicas, consideradas estágios avançados de doença, são cada vez mais raras, e a maioria dos indivíduos portadores de DC apresentam poucos sintomas, sutis e inespecíficos, o que requer do médico conhecimento e perspicácia para pensar no diagnóstico ${ }^{1}$.

Os indivíduos portadores de DC, não diagnosticados ou negligenciados, apresentam um maior risco de morbiletalidade a médio ou longo prazo ${ }^{2}$. Nos últimos anos, tem sido chamada a atenção para o fato de que a DC predispõe a anormalidades ósseas e a alterações no metabolismo do cálcio, resultando em osteomalácia, osteoporose e raquitis$\mathrm{mo}^{2-4}$. A patogênese da osteopenia não é completamente esclarecida, mas o desenvolvimento de hipocalcemia (por má absorção de cálcio) é provavelmente o evento central que poderá levar a outros distúrbios, particularmente aos níveis elevados de paratormônio e reabsorção óssea ${ }^{2}$.

Carvalho et al. ${ }^{5}$, em interessante artigo nesse número do Jornal de Pediatria, mostram uma redução na densidade mineral óssea em um grupo de adolescentes com diagnóstico de doença celíaca e em dieta isenta de glúten, a pelo menos um ano. Chama a atenção o fato de o diagnóstico ter sido realizado após os dois anos na maioria dos pacientes estudados, o que desperta a curiosidade para saber a forma de apresentação clínica desses pacientes e o tempo entre o início dos sintomas e o diagnóstico, a fim de se ter um melhor entendimento das repercussões da doença nas funções digestivo-absortivas. $\mathrm{O}$ fato do grupo de adolescentes celíacos apresentar uma diferença importante na média da

\footnotetext{
* Professora-adjunta de Pediatria, Universidade Federal de Pernambuco. Doutora em Pediatria, EPM/UNIFESP.
}

estatura em relação aos adolescentes do grupo controle sugere um agravo nutricional crônico, uma vez que o grupo de estudo foi semelhante em relação ao estadiamento puberal. Talvez a alteração observada em relação à densidade mineral óssea esteja associada a um retardo do diagnóstico, e, consequientemente, do início do tratamento. Portanto, nesse grupo de pacientes, é de grande importância que seja assegurada a adesão à dieta isenta de glúten, objetivando minimizar as alterações ósseas. Nas crianças não foram observadas diferenças em relação a esse parâmetro, mas é importante lembrar que o tamanho amostral não foi estimado para essa faixa etária, o que torna o resultado não conclusivo, uma vez que a amostra foi pequena.

Walters et al. ${ }^{6}$ observaram osteopenia em pacientes que não seguiam a dieta de isenção de glúten e naqueles com pouco tempo de tratamento, mas observaram densidade óssea normal naqueles que aderiam à dieta há pelo menos três anos.

A importância do estudo de Carvalho et al. ${ }^{5}$ reside no fato de chamar a atenção para a necessidade do diagnóstico precoce, assim como para a instituição da dieta isenta de glúten, com o objetivo de reduzir a morbidade da doença, especialmente quanto ao comprometimento da massa óssea. Considerando que a massa óssea é definida antes dos 20 anos, a identificação precoce de possíveis portadores de doença celíaca é de fundamental importância. Szathmári et al. ${ }^{3}$ não observaram diferença entre a densidade mineral óssea em um grupo de crianças e adolescentes celíacos em dieta isenta de glúten há mais de três anos, embora tenham observado repercussão no tamanho dos ossos.

Esses dados, somados a outras evidências, embasam a necessidade de se implementar em curto prazo uma triagem em nível populacional objetivando a identificação precoce da doença, pois assim reduziria a chance dos portadores de DC apresentarem morbidade residual. Por outro lado, a adesão à dieta é, à luz do conhecimento atual, a medida mais importante para assegurar mineralização óssea normal a crianças e adolescentes celíacos. 
Quando o diagnóstico da doença celíaca é feito em adultos, a adesão à dieta não garante a normalização da densidade mineral óssea, portanto essa seria uma população de risco para a ocorrência de fraturas. Recentemente, Thomason et al. ${ }^{7}$ estudaram 244 pacientes que tiveram o diagnóstico de DC na vida adulta e compararam a ocorrência de fraturas com 161 controles, e não observaram diferenças entre os dois grupos. Di Stefano et al. ${ }^{8}$ chamam a atenção para co-variáveis que precisam ser analisadas, quando se estuda a associação entre DC e osteopenia/ osteoporose em adultos, tais como sexo, atividade física, exposição ao sol, hábito de fumar e gravidade da doença. É certo que estudos de seguimento com pacientes nas diversas formas de apresentação clínica da doença, e que levem em consideração o tempo da doença antes do diagnóstico e se há adesão à dieta, irão trazer maiores subsídios para o entendimento do problema.

A indicação de dieta sem glúten por toda vida para os portadores da DC é um consenso internacional, embora nas formas ditas assintomáticas, seja, por vezes, questionada. Como as alterações histológicas do intestino delgado podem ser as mesmas daqueles com sintomas, assume-se que mesmo os indivíduos assintomáticos correm riscos em longo prazo, e devem ser colocados em dieta isenta de glúten ${ }^{9}$. Os pacientes portadores das formas silenciosas ou com sintomatologia discreta estão possivelmente sujeitos a um maior risco de agravos à saúde, devido à dificuldade do diagnóstico e ao retardo na introdução da dieta.

\section{Referências bibliográficas}

1. Brandt KG, Silva GAP. Doença celíaca. In: Ferreira CT, Carvalho E, Silva LR, editores. Gastroenterologia e Hepatologia em Pediatria. Diagnóstico e Tratamento. Rio de Janeiro: MEDSI; 2003.p.161-74.

2. Farrell RJ, Kelly CP. Celiac sprue. NEngl J Med 2002;346:180-8.

3. Szathmári M, Tulassay T, Arató A, Bodánszky H, Szabó A, Tulassay Z. Bone mineral content and density in asymptomatic children with coeliac disease on a gluten-free diet. Eur J Gastroenterol Hepatol 2001;13:419-24.

4. Kalayci AG, Kansu A, Girgin N, Kucuk O, Aras G. Bone mineral density and importance of a gluten-free diet in patients with celiac disease in childhood. Pediatrics [periódico online] 2001;108:89. Disponível: http://pediatrics.aappublications.org/ cgi/content/full/108/5/e89. Acessado: 18 de julho de 2003.

5. Carvalho CN, Sdepanian VL, Morais MB, Fagundes-Neto U. Doença celíaca em tratamento: avaliação da densidade mineral óssea. J Pediatr (Rio J) 2003;79:303-8.

6. Walters JR, Banks LM, Butcher GP, Fowler CR. Detection of low bone mineral density by dual energy $\mathrm{x}$ ray absorptiometry in unsuspected suboptimally treated coeliac disease. Gut 1995;37: 220-4.

7. Thomason K, West J, Logan RF, Coupland C, Holmes GK. Fracture experience of patients with coeliac disease: a population based survey. Gut 2003;52:518-22

8. Di Stefano M, Veneto G, Corrao G, Corazza GR. Role of lifestyle factors in the pathogenesis of osteopenia in adult coeliac disease: a multivariate analysis. Eur J Gastroenterol Hepatol 2000;12: 1195-9.

9. Hill ID, Bhatnagar S, Cameron DJ, De Rosa S, Maki M, Russell GJ, et al. Celiac Disease: Working Group Report of the First World Congress of Pediatric Gastroenterolgy, Hepatology, and Nutrition. J Pediatr Gastroenterol Nutr 2002;35 Suppl 2:78-88. 